Louisiana State University

LSU Digital Commons

$9-22-2016$

\title{
Pronounced Dielectric and Hydration/Dehydration Behaviors of Monopolar Poly(N-alkylglycine)s in Aqueous Solution
}

\author{
Kengo Arai \\ Tokyo University of Agriculture and Technology \\ Naoya Sagawa \\ Tokyo University of Agriculture and Technology \\ Toshiyuki Shikata \\ Tokyo University of Agriculture and Technology \\ Garrett L. Sternhagen \\ Louisiana State University \\ Xin Li \\ Louisiana State University
}

See next page for additional authors

Follow this and additional works at: https://digitalcommons.Isu.edu/chemistry_pubs

\section{Recommended Citation}

Arai, K., Sagawa, N., Shikata, T., Sternhagen, G., Li, X., Guo, L., Do, C., \& Zhang, D. (2016). Pronounced Dielectric and Hydration/Dehydration Behaviors of Monopolar Poly(N-alkylglycine)s in Aqueous Solution. Journal of Physical Chemistry B, 120 (37), 9978-9986. https://doi.org/10.1021/acs.jpcb.6b05379

This Article is brought to you for free and open access by the Department of Chemistry at LSU Digital Commons. It has been accepted for inclusion in Faculty Publications by an authorized administrator of LSU Digital Commons.

For more information, please contact ir@lsu.edu. 


\section{Authors}

Kengo Arai, Naoya Sagawa, Toshiyuki Shikata, Garrett L. Sternhagen, Xin Li, Li Guo, Changwoo Do, and Donghui Zhang 


\section{Pronounced Dielectric and Hydration/Dehydration}

\section{Behaviors of Monopolar Poly( $N$-alkylglycine $)$ s in}

\section{Aqueous Solution}

Kengo Arai ${ }^{1}$, Naoya Sagawa ${ }^{2}$, Toshiyuki Shikata ${ }^{* 1,2}$, Garrett L. Sternhagen ${ }^{3}$, Xin Li $^{3}{ }^{3}$ Li Guo, ${ }^{4}$ Changwoo Do ${ }^{5}$ and Donghui Zhang ${ }^{3}$

${ }^{1}$ Division of Natural Resources and Eco-materials, Graduate School of Agriculture, Tokyo University of Agriculture and Technology, 3-5-8 Saiwai-cho, Fuchu, Tokyo 183-8509, Japan

${ }^{2}$ Department of Symbiotic Science of Environment and Natural Resources, The United Graduate School of Agriculture, Tokyo University of Agriculture and Technology, 3-5-8 Saiwai-cho, Fuchu, Tokyo 183-8509, Japan

${ }^{3}$ Department of chemistry, Louisiana State University, Baton Rouge, Louisiana 70873, USA

${ }^{4}$ The Molecular Foundry, Lawrence Berkeley National laboratory, Berkeley, California 94720, USA

${ }^{5}$ Biology and Soft Matter Division, Neutron Sciences Directorate, Oak Ridge National Laboratory, Oak Ridge, TN 37831, USA

*To whom correspondence should be addressed. E-mail address: shikata@cc.tuat.ac.jp 
KEYWORDS: polyamide, poly( $N$-methylglycine), poly( $N$-ethylglycine), monopolar chain molecule, dielectric relaxation, Rouse model.

ABSTRACT: Poly $\left(N\right.$-methylglycine) $\left(\mathrm{NMG}_{n}\right)$ and poly( $N$-ethylglycine $)\left(\mathrm{NEG}_{n}\right)$ obtained by polymerization reaction initiated by benzylamine have no carboxy termini as normal polyamides, but have only amino termini, which are dissociative to be cationic ones in aqueous media at the $\mathrm{pH}$ value of ca 9.5 observed in aqueous solution without any buffer regents. Therefore, polypeptoids such as $\mathrm{NMG}_{n}$ and $\mathrm{NEG}_{n}$ possessing the degree of polymerization $(D P)$ higher than a certain value behave as cationic monopolar polymeric chain molecules in aqueous solution. It has not been clarified so far whether such a monopolar chain molecule exhibits dielectric relaxation (DR) behavior resulted from its molecular motions in aqueous media or not as dipolar chain molecules. Broad band DR measurements revealed that $\mathrm{NMG}_{19}$ and $\mathrm{NEG}_{17}$ possessing the $D P$ of 19 and 17, respectively, dissolved in pure water clearly demonstrate pronounced DR behavior caused by fluctuating molecular motions of cationic termini at relaxation times of ca 4 and $9 \mathrm{~ns}$ at $10{ }^{\circ} \mathrm{C}$. Hydration numbers of $\mathrm{NMG}_{19}$ and $\mathrm{NEG}_{17}$ per monomeric residue $\left(n_{\mathrm{m}}\right)$ in aqueous solution were also evaluated via DR data as functions of temperature, and the $n_{\mathrm{m}}$ value ca 4.5 at $10{ }^{\circ} \mathrm{C}$ showed a remarkable reducing down to ca 2.0 around 40 and $30^{\circ} \mathrm{C}$, respectively, depending on a difference in the substituted group: methyl and ethyl groups. This temperature dependent hydration/dehydration behavior found in $\mathrm{NMG}_{19}$ and $\mathrm{NEG}_{17}$ slightly influenced sizes and molecular dynamics of the monopolar chain molecules in aqueous solution.

\section{INTRODUCTION}


Molecular motions of polymeric chain molecules dissolved in viscous liquid media have been well understood based on a bead-spring model proposed by Rouse ${ }^{1}$ and modified by $\mathrm{Zimm}^{2}$ taking account of hydrodynamic interaction between beads constructing chains. ${ }^{3,4}$ Viscoelastic measurements are conventional methods to probe molecular motions of long polymeric chains like macromolecular molecules in solution in a frequency range lower than $10^{2} \mathrm{~s}^{-1}$. When the information of molecular motions in a frequency range higher than this value is necessary, dielectric relaxation (DR) experiments are useful methods to investigate molecular motions precisely. ${ }^{4}$ Especially, polymeric chain molecules bearing monomeric dipole moments aligned parallel along the polymer backbones, which are classified as type $A$ chain molecules, ${ }^{5,6}$ demonstrate pronounced DR related to fluctuation motions of chain termini, which are well described by a modified bead-spring model for the type A chain molecules. ${ }^{4}$ Polymeric chain molecules bearing dipolar, cationic and anionic, termini like usual polypeptides and protein molecules with carboxy and amino termini also behave as the type A chain in aqueous solution because of dissociation of both termini. ${ }^{5-9}$ Several years ago, DR measurements on aqueous solution of collagen model synthetic polyamide, ( $L$-proline- $L$-prolylglycine $)_{5}\left(\mathrm{PPG}_{5}\right)$, which bears carboxy and amino termini dissociative into a dipolar chain molecule in aqueous solution, in a frequency range higher than $10^{9} \mathrm{~s}^{-1}$ revealed that molecular motions of $\mathrm{PPG}_{5}$ are quantitatively described with the modified bead spring model. ${ }^{7-9}$

On the other hand, the modified bead spring model predicts that monopolar chain molecules bearing only a cationic or anionic group as a chain terminus also demonstrate DR behavior due to molecular motions governed by the Rouse and Zimm dynamics. ${ }^{5,6}$ However, the predicted magnitude of DR strength for the slowest relaxation mode of the monopolar chain molecule is one fourth of that of a dipolar chain molecule with the same degree of 
polymerization as the monopolar chain molecule. From a fundamental scientific point of view, whether such a monopolar polymeric chain molecule demonstrates profound DR behavior caused by fluctuating molecular motions of its monopolar terminus or not is a controversial open question, because there is no well-defined usual dipole moment responsible for the appearance of DR modes in the monopolar polymeric chain molecule. However, one should be aware that polypeptoid chains, such as $\mathrm{NMG}_{19}$ and $\mathrm{NEG}_{17}$, always bear amide groups generated by the polymerization process, which possess finite dipolar components parallel aligned to polymer chain backbones like the type A chain as schematically depicted in Scheme 1 below.

\section{-Scheme 1-}

At present, it is not difficult to synthesize monopolar polymeric chain molecules. For example, a polymerization process for polypeptoid molecule synthesis initiated by benzylamine (BzAM) is able to create monopolar polymeric chain molecules in aqueous solution, since BzAM has no ability to be an anionic chain terminus like a carboxy group. ${ }^{10,11}$ Then, employing BzAM initiated polypeptoid molecules dissolved in aqueous media as model molecules, one can investigate DR behavior of the monopolar polymeric chain molecule. In this study, broadband DR measurements were carried out on aqueous solutions of poly $(N$-methyl glycine $)$ and poly $(N-$ ethylglycine) possessing the degrees of polymerization $(D P)$ of $19\left(\mathrm{NMG}_{19}\right)$ and $17\left(\mathrm{NEG}_{17}\right)$, respectively, at several temperatures ranged from 10 to $70{ }^{\circ} \mathrm{C}$ to clarify whether DR processes resulted from the fluctuating molecular motions of chain termini or not in the case of monopolar polymeric chain molecules. Temperature dependencies of a hydration number per repeating residue, $n_{\mathrm{m}}$, were also determined for both $\mathrm{NMG}_{19}$ and $\mathrm{NEG}_{17}$ in aqueous solution. Moreover, the temperature dependency of molecular sizes was also discussed in relation to those of the hydration numbers for the polypeptoids. 


\section{EXPERIMENTAL}

Materials: $\mathrm{NMG}_{19}, \mathrm{NEG}_{17}$, and $\mathrm{NEG}_{18}$ were synthesized by ring-opening polymerization reaction of $N$-substituted $N$-carboxyanhydride monomer: $N$-methyl $N$-carboxyanhydride and $N$ ethyl $N$-carboxyanhydride, respectively. Procedures of monomer synthesis and ring-opening polymerization were reported in detail elsewhere ${ }^{11,12}$. Highly deionized water possessing specific electric resistance higher than $18 \mathrm{M} \Omega \mathrm{cm}$ obtained by a Direct-Q 3UV system (Millipore-Japan, Tokyo) was used as a solvent for sample preparation. The concentrations of $\mathrm{NMG}_{19}$ and $\mathrm{NEG}_{17}$ in the prepared aqueous solutions were ranged from $c=0.42$ to 1.0 and 0.1 to $0.41 \mathrm{M}$ in monomeric residue units, respectively. Deuterium oxide $\left(\mathrm{D}_{2} \mathrm{O}, 99.9\right)$ was purchased from SigmaAldrich and used as a solvent for sample preparation for small angle neutron scattering (SANS) measurements. The concentration of $\mathrm{NEG}_{18}$ in the prepared $\mathrm{D}_{2} \mathrm{O}$ solutions were $c=0.137 \mathrm{M}(1 \%$ in weight) in monomeric residue units.

Methods: A dielectric probe kit, 8507E, equipped with a network analyzer, N5230C, an ECal module N4693A, and performance probe 05 (Agilent Technologies, Santa Clara) was used for DR measurements over a frequency range from $50 \mathrm{MHz}$ to $50 \mathrm{GHz}\left(3.14 \times 10^{8} \sim 3.14 \times 10^{11} \mathrm{~s}^{-1}\right.$ in angular frequency $(\omega))$. The real and imaginary parts $\left(\varepsilon^{\prime}\right.$ and $\left.\varepsilon^{\prime \prime}\right)$ of the electric permittivity were automatically calculated from the reflection coefficients measured by the network analyzer via a program supplied by Agilent Technologies. A three-point calibration procedure using $n$-hexane, 3-pentanone and water as the standard materials was performed prior to all of the DR measurements. The details of the three-point calibration procedure used in this study have been 
described elsewhere ${ }^{12}$. DR measurements were performed at temperatures ranged from $T=10$ to $70{ }^{\circ} \mathrm{C}$ (accuracy of $\pm 0.1^{\circ} \mathrm{C}$ ) using a temperature controlling unit made of a Peltier device.

Density measurements for all of the aqueous sample solutions were carried out using a digital density meter, DMA4500 (Anton Paar, Graz), to determine the partial molar volumes of the solute molecules at the same temperatures used for the DR measurements.

The mean radius of gyration, $R_{\mathrm{G}}$, of $\mathrm{NEG}_{18}$ dissolved in aqueous $\left(\mathrm{D}_{2} \mathrm{O}\right)$ solution was obtained as functions of temperature, $T$, ranged from 10 to $70{ }^{\circ} \mathrm{C}$ using the standard small-angle neutron scattering (SANS) techniques. The SANS experiments were performed at EQ-SANS SNS in Oak Ridge National Laboratory. The scattering profiles of $\mathrm{NEG}_{18}$ were measured at EQSANS using the wavelength band of $0.424<\lambda<0.771 \mathrm{~nm}$ at $1.3 \mathrm{~m}$, with the $Q$ range covering $0.1-7.0 \mathrm{~nm}^{-1}$. The sample polymer, $\mathrm{NEG}_{18}$, was directly dissolved into deuterated water at the concentration of $1.0 \mathrm{wt} \%$ at room temperature, and then the solution sample was loaded into 2 $\mathrm{mm}$ thick banjo cell for measurements. Two sets of measurements were carried out: First run, the sample temperature was varied from 10 to $70{ }^{\circ} \mathrm{C}$ with a step of $10{ }^{\circ} \mathrm{C}$; second run, three measurements with doubled data acquisition time were performed at 10,40 and $70{ }^{\circ} \mathrm{C}$, respectively, to obtain better statistics at these three conditions. The transmission of the $\mathrm{NEG}_{18}$ solution is higher than 0.9 , indicating negligible multi-scattering events. The scattering intensities were scaled to absolute values on the basis of standard sample (Porsil B) measurement.

\section{RESULTS AND DISCUSSION}

Dielectric Behavior: Figure 1 shows the frequency dependence of $\varepsilon^{\prime}$ and $\varepsilon$ " of aqueous $\mathrm{NMG}_{19}$ at $c=0.5 \mathrm{M}$ and $10^{\circ} \mathrm{C}$ as a typical example. A large DR process found at $\omega=8 \times 10^{10} \mathrm{~s}^{-1}$ is easily assigned to the rotational relaxation mode of free water molecules, because only a DR 
process is observed at the same frequency in pure water. A small but significant DR process was additionally observed around $\omega=2 \times 10^{8} \mathrm{~s}^{-1}$, which will be attributed to DR process caused by fluctuating molecular motions of monopolar chain termini of $\mathrm{NMG}_{19}$ later. For quantitative relaxation analysis, the dielectric spectra seen in Figure 1 were decomposed into constituent fundamental Debye-type relaxation modes using formula given by eq 1 below

$$
\varepsilon^{\prime}=\sum_{j} \frac{\varepsilon_{j}}{1+\left(\omega \tau_{j}\right)^{2}}+\varepsilon_{\infty}, \varepsilon^{\prime \prime}=\sum_{j} \frac{\varepsilon_{j} \omega \tau_{j}}{1+\left(\omega \tau_{j}\right)^{2}}
$$

where $\varepsilon_{j}, \tau_{j}$ and $\varepsilon_{\infty}$ means DR strength and time for a mode $j$, and $\omega$ independent electric permittivity, respectively. ${ }^{7-9}$ Four Debye-type relaxation modes were necessary to reproduce the spectra data seen in Figure 1 perfectly as the best fit curves described with solid lines, and necessary constituent relaxation modes are shown in the same figure as broken lines. In all the aqueous samples prepared in this study, four Debye-type relaxation modes were necessary to reproduce experimental dielectric spectra as well as in the aqueous $\mathrm{NMG}_{19}$ solution at $c=0.5 \mathrm{M}$ seen in Figure 1.

-Figure 1-

The concentration, $c$, dependencies of $\tau_{j}$ and $\varepsilon_{j}$ determined via the curve fit procedure described above for an aqueous $\mathrm{NMG}_{19}$ system at $10{ }^{\circ} \mathrm{C}$ are shown in Figures 2 (a) and (b). We numbered $j=1$ from the fastest mode. In the case of the mode $j=1$, assignment was simply determined as a rotational relaxation mode of free water molecules in the aqueous $\mathrm{NMG}_{19}$ solution, because the relaxation time of the mode $j=1$ was identical to that of pure water (cf Figure 2 (a)). The DR strength, $\varepsilon_{1}$, which decreased almost proportionally to the concentration, $c$, as seen in Figure 2 (b), permitted one to determine hydration number, $n_{\mathrm{m}}$, per monomeric residue 
as described in the next section. The second fastest relaxation mode $j=2$ was assigned to the exchange mode of hydrated water molecules, because the relaxation strength, $\varepsilon_{2}$, was proportional to $c$ and was reasonably identical to a relaxation magnitude calculated from the hydration number, $n_{\mathrm{m}}$, determined from the $\varepsilon_{1}$ value assuming water molecules keep the same DR strength irrespective of the formation of hydrogen bonds due to the hydration behavior.

-Figures 2(a) and (b)-

The fourth, longest relaxation mode $j=4$ was assigned to the fluctuating molecular motion of cationically charged termini, $-\mathrm{N}^{+}\left(-\mathrm{CH}_{3}\right) \mathrm{H}_{2}$, of chain like $\mathrm{NMG}_{19}$ molecules, because the addition of aqueous sodium hydroxide $(\mathrm{NaOH})$ solution to promote the deprotonation of cationically charged termini substantially increased the relaxation mode $j=4$ as seen in Figure SI1 in Supporting Information. The $\mathrm{pH}$ values of aqueous $\mathrm{NMG}_{19}$ and $\mathrm{NEG}_{17}$ solution without any buffer chemicals were of $9.2-9.5$. This observation suggests that the degree of protonation of the polypeptoid chain molecules in aqueous solution is higher than 0.9 , since the typical $\mathrm{p} K_{\mathrm{a}}$ value of the secondary amine termini is $\sim 11 .{ }^{13}$ Because the alternation of electric charge condition of polypeptoid chain termini influenced the relaxation strength of the mode $j=4$, this mode corresponds to fluctuating molecular motions of polymer chains. Moreover, aqueous solution of collagen model polypeptide, $\mathrm{PPG}_{5}$, with a similar $D P=15$ (in residue numbers) also demonstrates pronounced $\mathrm{DR}$ at a frequency close to that for aqueous $\mathrm{NMG}_{19}$ solution: $\omega=$ $2 \times 10^{8} \mathrm{~s}^{-1}$ (cf Figures 1 and 2(a)), which has been confirmed to be the fluctuating molecular motion of dipolar termini of chain molecules, $\mathrm{PPG}_{5}{ }^{7-9}$ This experimental fact strongly reveals that the DR process found as the relaxation model $j=4$ results from the fluctuating molecular motion of monopolar polymeric chains of $\mathrm{NMG}_{19}$. Furthermore, the dependence of $\varepsilon_{4}$ in proportion to $c$ recognized in Figure 2(b) is consistent with the assignment. 
Two kinds of conceivable explanations are possible for the smallest relaxation process observed as the relaxation mode $j=3$ with the relaxation time of $\tau_{3}=90 \mathrm{ps}$. The first possible assignment for this relaxation process is the (first) overtone of fluctuating molecular motion of polymeric chain molecules, $\mathrm{NMG}_{19}$. The other, second assignment is molecular motion of monomeric residues, which possess a amide group, $-\mathrm{N}\left(-\mathrm{CH}_{3}\right) \mathrm{C}(=\mathrm{O})$-, bearing a relatively large dipole moment generated by the polymerization process. This molecular motion corresponds to the fastest dynamics occurring in the polymeric chain via rotation around chemical bonds. In a later section, the second assignment will be accepted as an essential mechanism for the relaxation mode $j=3$.

In the case of an aqueous $\mathrm{NEG}_{17}$ system, similar DR spectra to those of the aqueous $\mathrm{NMG}_{19}$ system (cf Figure 1) were observed. The same curve fit procedure was employed also in the aqueous $\mathrm{NEG}_{17}$ system to decompose DR spectra into constituent Debye-type relaxation components as used in the aqueous $\mathrm{NMG}_{19}$ system above. Dielectric parameters necessary to reproduce the obtained DR spectra for the aqueous $\mathrm{NEG}_{17}$ system at $10^{\circ} \mathrm{C}$ are shown as functions of the concentration of $\mathrm{NEG}_{17}, c$, in Figures 3(a) and (b). Since the dependencies of parameters for the modes $j=1$ and 2 on $c$ in the aqueous $\mathrm{NEG}_{17}$ system well agree with those observed in the aqueous $\mathrm{NMG}_{19}$ system, the fundamental mechanisms for the modes: the rotational relaxation of free water molecules $(j=1)$ and the exchange process of water molecules hydrated to $\mathrm{NEG}_{19}$ molecules $(j=2)$, are not so much influenced by a difference in the monomer species of the polymeric chain molecules. On the other hand, although the $c$ dependencies of $\varepsilon_{3}$ and $\varepsilon_{4}$ for the aqueous $\mathrm{NEG}_{17}$ system were similar to those for the aqueous $\mathrm{NMG}_{19}$ system, the $\tau_{4}$ value was almost twice as long as that of the aqueous $\mathrm{NMG}_{19}$ system. Then, one might conclude 
that the mechanisms for the mode $j=4$ is highly influenced by the difference in the chemical species of monomers.

-Figures 3(a) and (b)-

Hydration Number: The dependencies of $\varepsilon_{1}$ and the partial molar volume per monomeric residue $\left(V_{\mathrm{m}}\right)$ on $c$ allow us to determine the hydration number, $n_{\mathrm{m}}$, using eq 2 given below ${ }^{7-9}$

$$
\frac{\varepsilon_{1}}{\varepsilon_{\mathrm{w}}}=\frac{1-10^{-3} V_{\mathrm{m}} c}{1+10^{-3} V_{\mathrm{m}} c / 2}-10^{-3} V_{\mathrm{w}} c n_{\mathrm{m}}
$$

where $V_{\mathrm{W}}$ represents the partial molar volume of water molecules. Temperature, $T$, dependence of $n_{\mathrm{m}}$ for aqueous $\mathrm{NMG}_{19}$ and $\mathrm{NEG}_{17}$ systems are shown in Figures $4(\mathrm{a})$ and (b), respectively. Pronounced dehydration phenomena, which mean decreasing in the $n_{\mathrm{m}}$ value with increasing $T$, were clearly recognized in both the aqueous systems. However, dehydration temperature, $T_{\mathrm{dh}}$, defined as a middle point between asymptotic hydration numbers at low, $n_{\mathrm{mL}}$, and high temperature, $n_{\mathrm{mH}}$, differs depending on the chemical species of monomeric residue, ie. $T_{\mathrm{dh}} \sim 40$ and $30{ }^{\circ} \mathrm{C}$ for $\mathrm{NMG}_{19}$ and $\mathrm{NEG}_{17}$, respectively, as seen in Figure 4 .

-Figure 4-

The values of $n_{\mathrm{mL}} \sim 4.5$ and $n_{\mathrm{mH}} \sim 2$ look less dependent on the chemical species of monomeric residue (cf Figure 4). It is worthy to note that the values of $n_{\mathrm{mL}} \sim 9, n_{\mathrm{mH}} \sim 3$ and $T_{\mathrm{dh}}$ $\sim 27^{\circ} \mathrm{C}$ for $\mathrm{PPG}_{5},{ }^{9}$ the asymptotic hydration number, $n_{\mathrm{mL}}$, for $\mathrm{PPG}_{5}$ is about twice as large as those of $\mathrm{NMG}_{19}$ and $\mathrm{NEG}_{17}$. These observations strongly propose that the values of $n_{\mathrm{mL}}$ and $n_{\mathrm{mH}}$ essentially depend on the chemical species of monomeric residue, whereas a difference between 
methyl and ethyl group as substitute ones is small and does not make difference in the values of $n_{\mathrm{mL}}$ and $n_{\mathrm{mH}}$.

The hydration/dehydration behavior observed in aqueous $\mathrm{NMG}_{19}$ and $\mathrm{NEG}_{17}$ systems as a function of temperature very slightly influenced the sizes of polymeric chains in aqueous solution. Figure 5 demonstrates temperature, $T$, dependence of the mean radius of gyration, $R_{\mathrm{G}}$, for $\mathrm{NEG}_{18}$ obtained from SANS measurements in aqueous $\left(\mathrm{D}_{2} \mathrm{O}\right)$ solution via the simplest analysis method proposed by Guinier ${ }^{14}$. Dependencies of the logarithmic values of macroscopic cross section (scattering intensity, $I(Q)$ ) on the square of the magnitude of a scattering vector, $Q^{2}$, for aqueous $\mathrm{NEG}_{18}$ solution determined at several temperatures ranged from 10 to $70{ }^{\circ} \mathrm{C}$ were analyzed as described in Supporting Information. The incoherent scattering component $\left(I_{\text {inc }}\right)$ was determined from the data fitting procedure using a flexible cylinder chain model ${ }^{15}$ involving an incoherent background component as a fit parameter. After the subtraction of the incoherent background, $I_{\mathrm{inc}}$, the coherent scattering can be analyzed using Guinier Plot to obtain $R_{\mathrm{g}}$. In the procedure, points from the 5 th to 25 th data in a range of $Q<1.0 \mathrm{~nm}^{-1}$ were used to precisely determine the initial slope between $\ln \left\{l(Q)-I_{\text {inc }}\right\}$ and $Q^{2}$. Limited by the current instrument ability, the statistical error of $R_{\mathrm{g}}$, as shown in Figure 5, is relatively large compared to the variation of $R_{\mathrm{g}}$ values. Although the determined $R_{\mathrm{g}}$ data were highly scattered, the fact that the values of $R_{\mathrm{G}}$ for $\mathrm{NEG}_{18}$ molecules slightly increased with increasing $T$, at least did not decrease with increasing $T$, as observed in Figure 5 reveals that the dehydration behavior in monomeric residues does not reduce the whole chain sizes in aqueous systems of $\mathrm{NEG}_{17}$. This weak $T$ dependence of molecular sizes induced by the dehydration process found in $\mathrm{NEG}_{17}$ is clearly opposite to that observed in aqueous $\mathrm{PPG}_{5}$ system ${ }^{9}$, in which the mean molecular sizes of $\mathrm{PPG}_{5}$ clearly decreases with increasing $T$. Significant differences observed in the asymptotic hydration 
numbers, $n_{\mathrm{mL}}$ and $n_{\mathrm{mH}}$, between $\mathrm{PPG}_{5}\left(n_{\mathrm{mL}} \sim 9 \text { and } n_{\mathrm{mH}} \sim 3\right)^{9}$ and $\mathrm{NEG}_{17}$ would be a reason for the oppositive $T$ dependence in the sizes of polymer chains. Because the average monomeric residue of $\mathrm{PPG}_{5}$ holds hydrated water molecules twice as many as that of $\mathrm{NRG}_{17}$, the amount of dehydrated water molecules is also twice as many. Then, the dehydration process of $\mathrm{PPG}_{5}$ in aqueous solution possibly induces a notable shrinkage in size.

-Figure 5-

Dynamics of Polymeric Chains: According to a modified bead-spring model proposed by Stockmayer et $\mathrm{al}^{5,6}$, the mean square of dipole moment, $<\left(\mu_{\mathrm{p}}^{\mathrm{bs}}\right)^{2}>$, and relaxation time, $\tau_{p}$ bs , for a fundamental mode number $p$ are given by eqs 3 and 4 .

$$
\begin{aligned}
& \left\langle\left(\mu_{p}^{\mathrm{bs}}\right)^{2}\right\rangle=\frac{2(N-1) b^{2} e^{2}\left(q^{i} \pm q^{\mathrm{f}}\right)^{2}}{\pi^{2} p^{2}},+; \text { odd and -; even } \\
& \tau_{p}^{\mathrm{bs}}=\frac{b^{2} \zeta}{12 k_{\mathrm{B}} T \sin ^{2}\left(\frac{\pi p}{2 N}\right)}
\end{aligned}
$$

where $N, \zeta, b, e, q_{\mathrm{i}}$ and $q_{\mathrm{f}}$ represents the number of beads in a chain molecule, friction coefficient of a bead, length of a spring, elementary electric charge, degree of ionization of the initial bead and degree of ionization of the final bead, respectively. Dipolar polymeric chain molecules possess nonzero $q_{\mathrm{i}}$ and $q_{\mathrm{f}}$ values. However, in the case of monopolar chain molecules, $q_{\mathrm{i}}$ or $q_{\mathrm{f}}$ should be zero. When one compares the magnitudes of $\left\langle\left(\mu_{1}{ }^{\text {bs }}\right)^{2}>\right.$ at the mode of $p=1$ (corresponding to the mode $j=4$ in experimental results) for a monopolar $\left(q_{\mathrm{i}}=1\right.$ and $\left.q_{\mathrm{f}}=0\right)$ to that of dipolar $\left(q_{\mathrm{i}}=q_{\mathrm{f}}=1\right)$ chain molecules bearing the same parameters: $N, \zeta$ and $b$, the predicted value for the monopolar chain molecule is one fourth of that of the dipolar one. It is worthy to 
note that even in the case of monopolar polymeric chain molecules without well-defined usual dipole moments, finite non-zero $<\left(\mu_{1} \text { bs }\right)^{2}>$ values are predicted by the modified bead-spring $\operatorname{model}^{5,6}$.

Because the magnitude of DR strength is proportional to the quantity $c<\left(\mu_{1}^{\text {bs }}\right)^{2}>\left(k_{\mathrm{B}} T\right)^{-1}$, where $k_{\mathrm{B}}$ means the Boltzmann constant, from an experimental point of view, the concentration reduced magnitude, $\varepsilon_{4} c^{-1}$, for the slowest mode $j=4$ should be compared with each other between monopolar and dipolar polymeric chains which are made from the same monomeric species and possess identical $D P$ values. However, since we do not have dipolar $\mathrm{NMG}_{19}$ and $\mathrm{NEG}_{17}$ samples at present, such a quantitative comparison should be performed in the near future using systematically synthesized samples. The $\varepsilon_{4} c^{-1}$ data of monopolar $\mathrm{NMG}_{19}$ and $\mathrm{NEG}_{17}$ might be roughly compared to that of $\mathrm{PPG}_{5}$ possessing a similar $\tau_{4}$ value to the $\mathrm{NMG}_{19}$ and $\mathrm{NEG}_{17}$, which means the size of $\mathrm{PPG}_{5}$ in aqueous solution is not so different from those of the $\mathrm{NMG}_{19}$ and $\mathrm{NEG}_{17}$.

The temperature dependence of the $\varepsilon_{4} c^{-1} T$ values of $\mathrm{NMG}_{19}$ and $\mathrm{NEG}_{17}$ are shown in Figure 6. The $\varepsilon_{4} c^{-1} T$ of $\mathrm{NMG}_{19}$ and $\mathrm{NEG}_{17}$ seem to be $1.3 \times 10^{3} \mathrm{M}^{-1} \mathrm{~K}$ at $T=20{ }^{\circ} \mathrm{C}$ and is about one tenth of the value of PPG5 at the same temperature. Since this observation obviously disagrees with the prediction via eq 3: one fourth, it is likely that the modified dead-spring model does predict an incorrect dipole moment value in the case of the monopolar polymeric chain molecules. Since no theoretical models including the modified bead-spring model are able to predict DR behavior of monopolar polymeric chain molecules correctly at present, a new model that holds well in both the mono- and dipolar molecules should be proposed in the near future. A slight increase observed in the $\varepsilon_{4} c^{-1} T$ of $\mathrm{NMG}_{19}$ and $\mathrm{NEG}_{17}$ with increasing $T$ suggests limited increasing in their sizes corresponding to the behavior found in Figure 5, whereas the $\varepsilon_{4} c^{-1} T$ of 
$\mathrm{PPG}_{5}$ markedly decreases with increasing temperature ${ }^{9}$. This $T$ dependent change in the $\varepsilon_{4} \mathrm{C}^{-1} T$ value, which is correlated with the $T$ dependence of sizes of $\mathrm{NMG}_{19}$ and $\mathrm{NEG}_{17}$ in aqueous solution, strongly reveals that the mode $j=4$ corresponds to the fluctuating molecular motions of chain termini of the monopolar polypeptides.

-Figure 6-

The assignment for the mode $j=3$ from the viewpoint of dynamical behavior is not simple. If one accepts the eqs 3 and 4 and substitutes $q_{\mathrm{i}}=0$ as the constraint condition, an overtone of a bead-spring model chain designated by the mode number $p=2$ would be observed as the experimental mode $j=3$. In this case, observable relaxation strength as the mode $j=3$ would be one fourth of that of the mode $j=4$, and is substantially smaller than the observed ratio of $\varepsilon_{3} \varepsilon_{4}^{-1} \sim 0.6$ for both the aqueous $\mathrm{NMG}_{19}$ and $\mathrm{NEG}_{17}$ systems as seen in Figures 2(b) and 3(b). From these considerations, it is not likely that the mode $j=3$ is attributed to the overtone designated by the mode number $p=2$. Consequently, the mode $j=3$ is assigned to the fundamental molecular motion of monomeric residues bearing finite dipole moments.

Temperature, $T$, dependence of each relaxation time, $\tau_{j}$, for the aqueous $\mathrm{NMG}_{19}$ and $\mathrm{NEG}_{17}$ systems are shown in Figures 7(a) and (b). The slopes of lines drawn in these figures mean apparent activation energies for each DR mode observed. The activation energy of the mode $j=1$ simply means that of the rotational relaxation mode of free water molecules; $E_{1}{ }^{*}=19$ $\mathrm{kJmol}^{-1}$. The evaluated activation energy for the mode $j=2, E_{2}{ }^{*}=23 \mathrm{kJmol}^{-1}$, slightly higher than the value of $E_{1}{ }^{*}$, suggests the contribution of an additional activation energy related to the hydration energy of water molecules to the peptoid molecules. ${ }^{16}$ Plots of $\tau_{3}$ vs $T^{-1}$ seen in Figures 7(a) and (b) do show greater slopes than that of the modes $j=1$. This observation suggests that 
dynamics of the modes $j=3$ is not controlled by the activation energy of free water molecules of $19 \mathrm{kJmol}^{-1}$, but by other $T$ dependent parameters relating to the dynamics of apparent dipole moments such as effective hydrodynamic sizes (volumes) of monomeric units.

In the case of the mode $j=4$, the obtained value of the apparent activation energy, $E_{4}{ }^{*}$, seem to be incidentally identical to $E_{1}{ }^{*}=19 \mathrm{kJmol}^{-1}$ and markedly smaller than that of $E_{3} *$ in both the aqueous $\mathrm{NMG}_{19}$ and $\mathrm{NEG}_{17}$ systems as seen in Figures 7(a) and (b). If the mode $j=3$ is the overtone of the mode $j=4$ controlled by the same dynamics, the activation energies for the modes should be identical to each other. This is another reason why the mode $j=3$ is assigned to molecular motion of monomeric residues, not to the overtone of the mode $j=4$. It is possible that hydration/dehydration behavior seen in Figure 4 governs the $T$ dependencies of $\tau_{3}$ and $\tau_{4}$ values.

-Figures 7(a) and (b)-

As described above, the addition of $\mathrm{NaOH}$ into aqueous $\mathrm{NEG}_{17}$ solution to reduce the degree of protonation of amine termini substantially increased the $\varepsilon_{4}$ value. The magnitude of the mode $j=4$ approached to a certain value after the enough amount of $\mathrm{NaOH}$ addition (Figure SI1 in Supporting Information). Then, one might conclude that the degree of protonation of terminal amine termini markedly influences the value of apparent dipole moments corresponding to the mode $j=4$. As the result of the polymerization process, amide groups: $-\mathrm{N}\left(-\mathrm{CH}_{3}\right) \mathrm{C}(=\mathrm{O})$ - and $-\mathrm{N}(-$ $\left.\mathrm{CH}_{2} \mathrm{CH}_{3}\right) \mathrm{C}(=\mathrm{O})$-, bearing relatively large monomeric dipole moments, $\boldsymbol{\mu}_{\mathrm{m}}$, are generated in each polymeric chain molecule. Because the direction of $\boldsymbol{\mu}_{\mathrm{m}}$ for an amide group is not (perfectly) perpendicular to the polymer chain backbone as schematically depicted in Scheme 1, finite dipole components: parallel and perpendicular to the polymer backbone, $\mu_{\mathrm{m} / /}$ and $\mu_{\mathrm{m} \perp}$, are resulted from $\boldsymbol{\mu}_{\mathrm{m}}$ in each monomeric residue. Because the parallel component $\mu_{\mathrm{m} / /}$ for each 
monomer orientates along the polymer backbone, both of $\mathrm{NMG}_{19}$ and $\mathrm{NEG}_{17}$ are classified as monopolar type A chain molecules. Then, the summation of $\mu_{\mathrm{m} / /}$ over all the constituent monomeric residues would be the intrinsic dipole moment for each polymer chain, which results in the dielectric mode $j=4$. Consequently, it is possible that characteristics as the type A chain are the essential reason for the fluctuating molecular motions of polymer chain termini dielectrically observed as the mode $j=4$. On the other hand, time dependent fluctuation of $\mu_{\mathrm{m} \perp}$ resulted from monomeric molecular motion, the rotation around chemical bonds, should be the essential origin for the mode $j=3$. It is likely that the presence of a protonated amino terminus bearing a cationic electric charge effectively disturbs a (fundamental) dipole moment, $\boldsymbol{\mu}_{\mathrm{m}}$, of the neighbor residue as schematically represented in Scheme 1 due to the appearance of an additional terminus dipole moment, $\boldsymbol{\mu}_{\text {term. }}$. The alternation of the degree of protonation controlled by the addition of $\mathrm{NaOH}$ possibly influences the magnitude of the total dipole moment corresponding to the DR strength for the modes $j=3$ and 4 .

\section{CONCLUSIONS}

Dielectric behavior of aqueous solution of $\operatorname{Poly}(N$-methylglycine) at the degree of polymerization of $D P=19\left(\mathrm{NMG}_{19}\right)$ and $\operatorname{poly}(N$-ethylglycine $)$ at $D P=17\left(\mathrm{NEG}_{17}\right)$ possessing no carboxy terminus, but only an amino one, which is dissociative into a cationic charge terminus in the aqueous condition without any buffer chemicals. It was newly discovered that such electrically monopolar polymeric chain molecules, possessing finite monomeric dipolar components provided by amide groups aligned parallel to the polymer chain backbones, obviously exhibit pronounced dielectric relaxation behavior evidently resulted from their fluctuating molecular motions in aqueous media. The obtained dielectric behavior of aqueous $\mathrm{NMG}_{19}$ and $\mathrm{NEG}_{17}$ systems also permitted us to determine the temperature dependence of 
hydration numbers per monomeric residue in aqueous solution. A hydration number of ca 4.5 determined at $10{ }^{\circ} \mathrm{C}$ remarkably decreased down to ca 2 around 40 and $30{ }^{\circ} \mathrm{C}$, respectively, for $\mathrm{NMG}_{19}$ and $\mathrm{NEG}_{17}$. This temperature dependent hydration/dehydration behavior slightly influences sizes and molecular dynamics of the polymeric chain molecules in aqueous solution. ACKNOWLEDGMENTS: This work was partially supported by JSPS KAKENHI Grant-inAid for Scientific Research (B) Number 26288055. The SANS studies are supported by the U.S. Department of Energy under EPSCoR Grant DESC0012432 with additional support from the Louisiana Board of Regents. The Research at Oak Ridge National Laboratory's Spallation Neutron Source was sponsored by the Scientific User Facilities Division, Office of Basic Energy Sciences, U.S. Department of Energy.

Supporting Information: Effects of $\mathrm{NaOH}$ Addition on Dielectric Behavior and Temperature Dependence of SANS Data. This material is available free of charge via the Internet at http://pubs.acs.org. 


\section{REFERENCES}

1) Rouse, P. E. J. Chem. Phys. 1953, 21, 1272-1280.

2) Zimm, B. H. J. Chem. Phys. 1956, 24, 269-278.

3) Doi, M.; Edwards, S. F. The Theory of Polymer Physics; Oxford Scientific Publication: Oxford, 1986; Chapter 4.

4) Watanabe, H. Prog. Polym. Sci. 1999, 24, 1253-1403.

5) Stockmayer, W. H.; Baur, M. E. J. Am. Chem. Soc. 1964, 86, 3485-3489.

6) Stockmayer, W. H. Pure Appl. Chem. 1967, 15, 539-554.

7) Shikata, T.; Yoshida, N.; Minakawa, A.; Okuyama, K.; J. Phys. Chem. B 2009, 113, 90559058.

8) Shikata, T.; Minakawa,; Yoshida, N. A.; Okuyama, K.; J. Phys. Chem. B 2009, 113, 1450414512.

9) Shikata, T.; Yoshida, N.; Okuyama, K.; Phys. Chem. Lett. 2010, 1, 412-416.

10) Reyes, F. T.; Guo, L.; Hedgepeth, J. W.; Zhang, D.; Kelland, M. A. Energy Fuels 2014, 28, 6889-6896.

11) Hörz, C; Birke, A.; Kaps, L.; Decker, S.; Wächtersbach, E.; Fisher, K.; Schuppan, D.; Barz, M.; Schmidt, M. Macromolecules 2015, 48, 2074-2086.

12) Shikata, T.; Sugimoto, N. Phys. Chem. Chem. Phys. 2011, 13, 16542-16547. 
13) Smith, M. B.; March, J. March’s Advanced Organic Chemistry Reactions, Mechanisms, and Structure, 6th Ed.; Wiley: New Jersey, 2007; Chap. 8.

14) Guinier, A; Fournet, G. Small Angle Scattering of X-Rays, Trans. Wilson, C. B.; New York; Wiley: 1955.

15) Pedersen, J. S.; Schurtenberger, P. Macromolecules1996, 29, 7602-7612.

16) Ono, Y.; Shikata, T.; J. Chem. Soc. Am. 2006, 128, 10030-10031. 
Legend for Scheme 1. Schematic depiction showing the presence of dipolar components parallel and perpendicular $\left(\mu_{\mathrm{m} / /}\right.$ and $\left.\mu_{\mathrm{m} \perp}\right)$ to the polymer backbone resulted from monomeric dipole moments $\left(\boldsymbol{\mu}_{\mathrm{m}}\right)$ of amide groups generated by the polymerization process. The presence of cationic electric charge likely induces a new terminus dipole moment, $\boldsymbol{\mu}_{\text {term }}$, which disturbs neighbor residual dipole moment, $\boldsymbol{\mu}_{\mathrm{m}}$.

\section{Figure Captions}

Figure 1. The frequency $(\omega)$ dependence of real and imaginary parts of electric permittivity $(\varepsilon$ and $\left.\varepsilon^{\prime \prime}\right)$ of aqueous $\mathrm{NMG}_{19}$ solution at $c=0.5 \mathrm{M}$ and $10{ }^{\circ} \mathrm{C}$ as a typical example.

Figure 2. The concentration (c) dependencies of $\tau_{j}$ (a) and $\varepsilon_{j}$ (b) determined via curve fit procedure using eq 1 described in the text for an aqueous $\mathrm{NMG}_{19}$ system at $10^{\circ} \mathrm{C}$.

Figure 3. The concentration, $c$, dependencies of $\tau_{j}$ (a) and $\varepsilon_{j}$ (b) determined via curve fit procedure for an aqueous $\mathrm{NEG}_{17}$ system at $10{ }^{\circ} \mathrm{C}$.

Figure 4. Temperature $(T)$ dependencies of hydration numbers per monomeric residue $\left(n_{\mathrm{m}}\right)$ for aqueous $\mathrm{NMG}_{19}$ (a) and $\mathrm{NEG}_{17}$ (b) systems. Arrows indicate dehydration temperatures $\left(T_{\mathrm{dh}}\right.$; see text). 
Figure 5. $T$ dependence of the mean radius of gyration, $R_{\mathrm{G}}$, for $\mathrm{NEG}_{18}$ in aqueous (deuterium oxide: $\mathrm{D}_{2} \mathrm{O}$ ) solution resulted from SANS experiments via the simplest analysis method proposed by Guinier ${ }^{14}$. Large and small symbols represent the first and second run, respectively.

Figure 6. $T$ dependence of the $\varepsilon_{4} c^{-1} T$ values of $\mathrm{NMG}_{19}$ and $\mathrm{NEG}_{17}$ in aqueous solution.

Figure 7. The dependence of relaxation times $\tau_{2}$ and $\tau_{3}$ on $T^{-1}$ for the aqueous $\mathrm{NMG}_{19}(\mathrm{a})$ and $\mathrm{NEG}_{17}$ (b) systems. 
Scheme and Figures

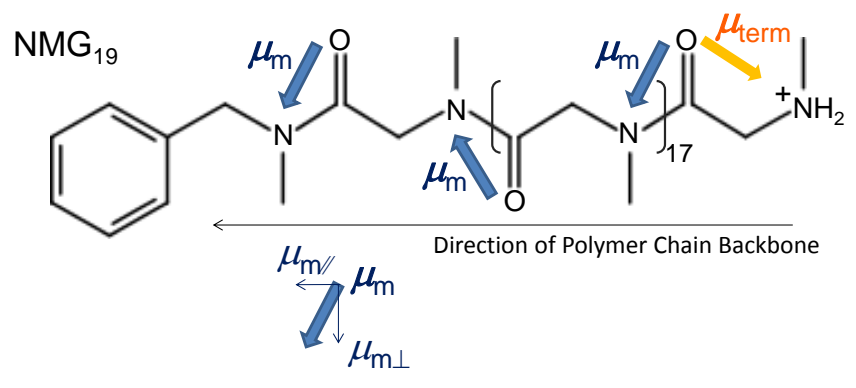

Scheme 1

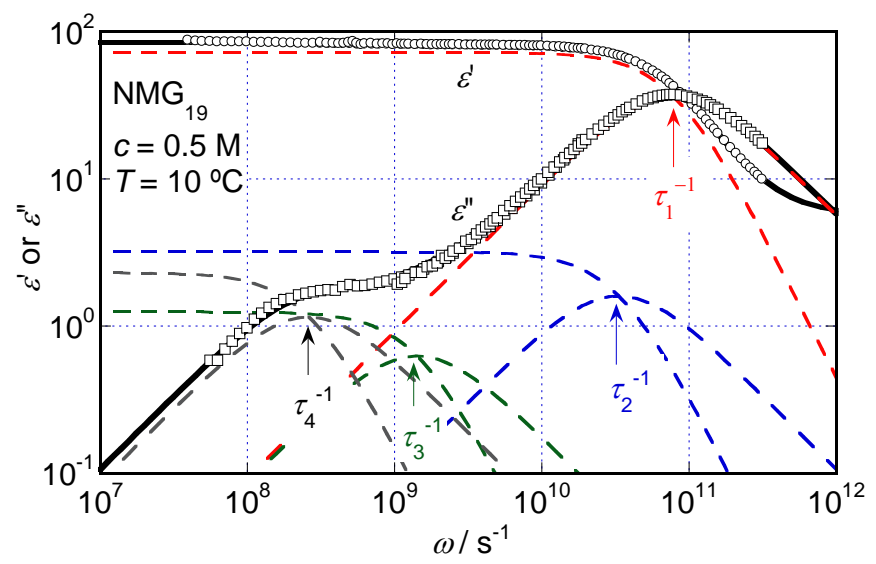

Figure 1
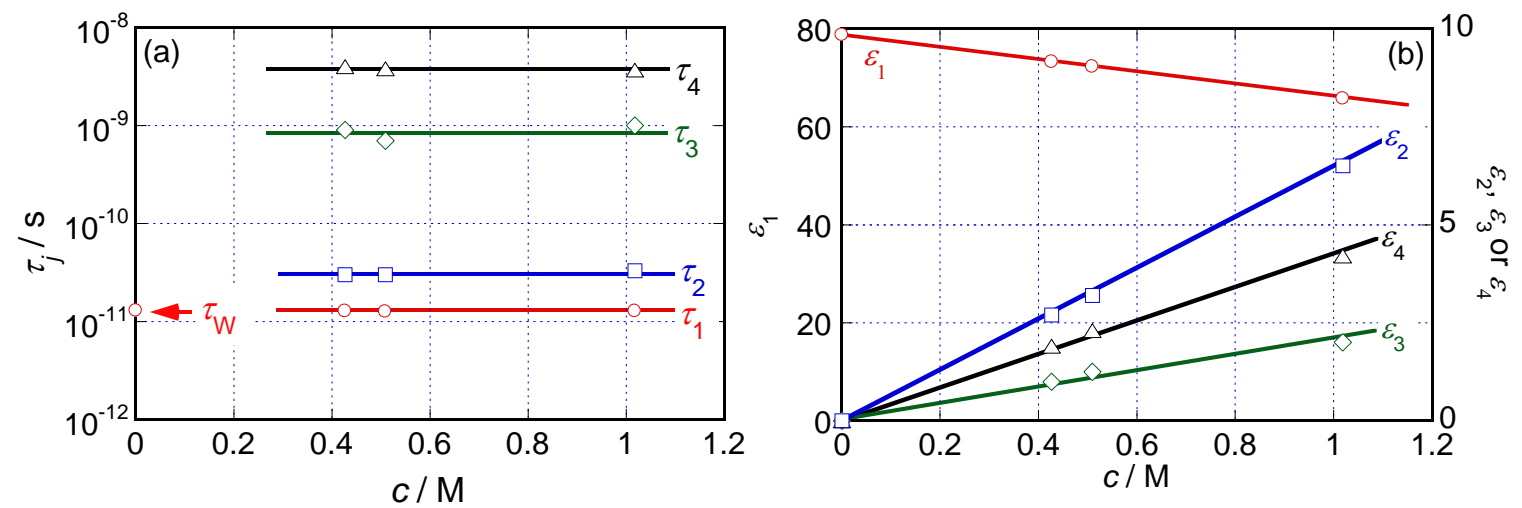

Figure 2 

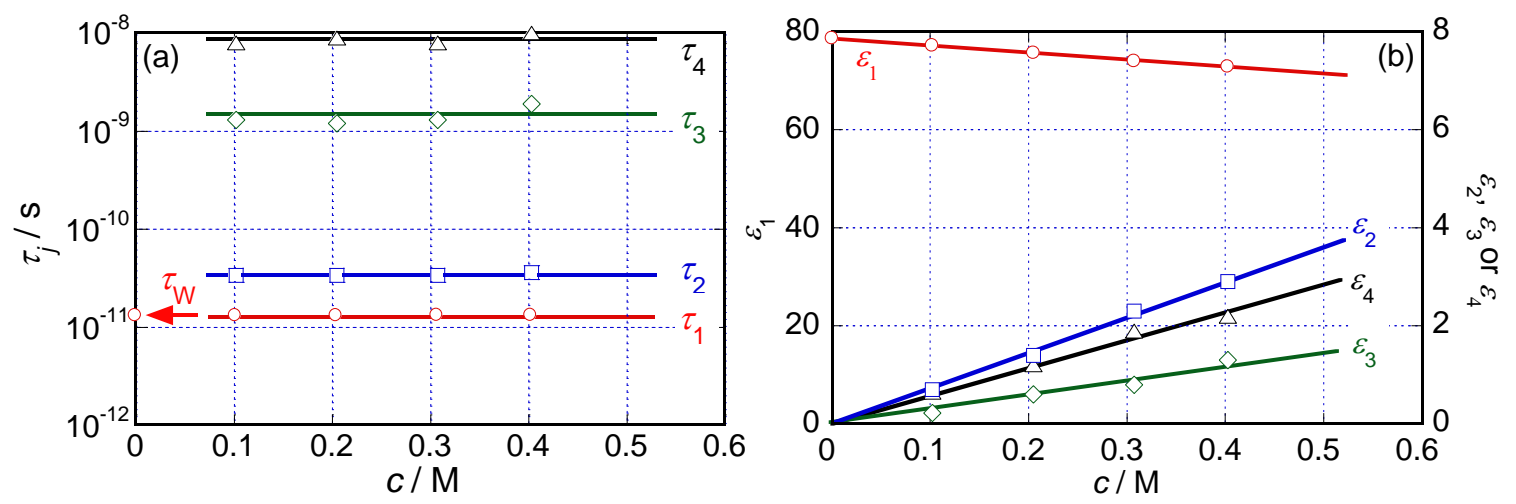

Figure 3
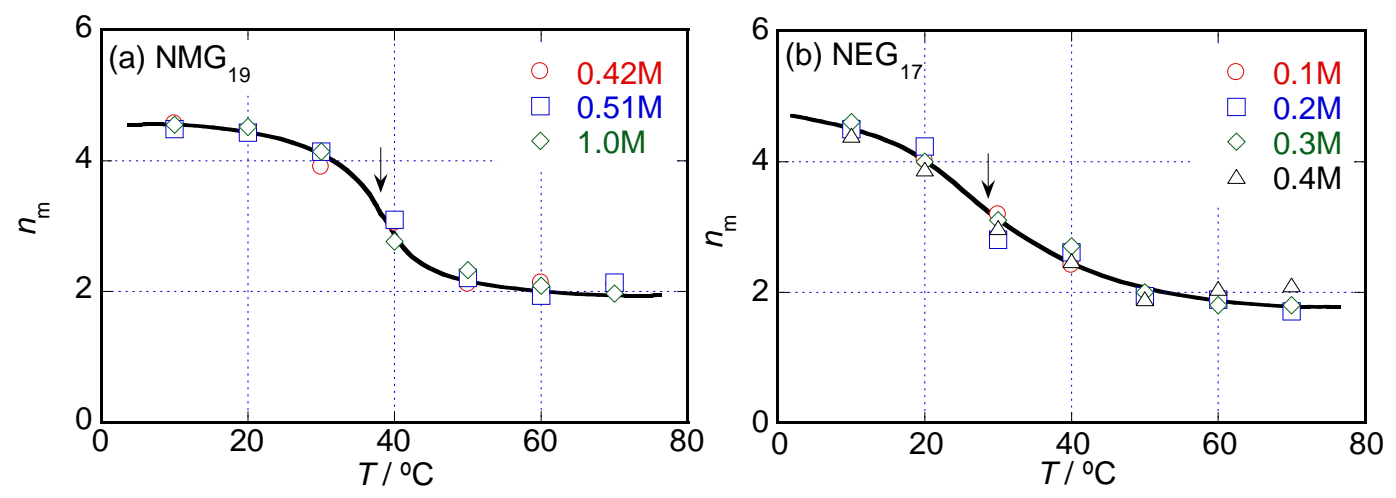

Figure 4

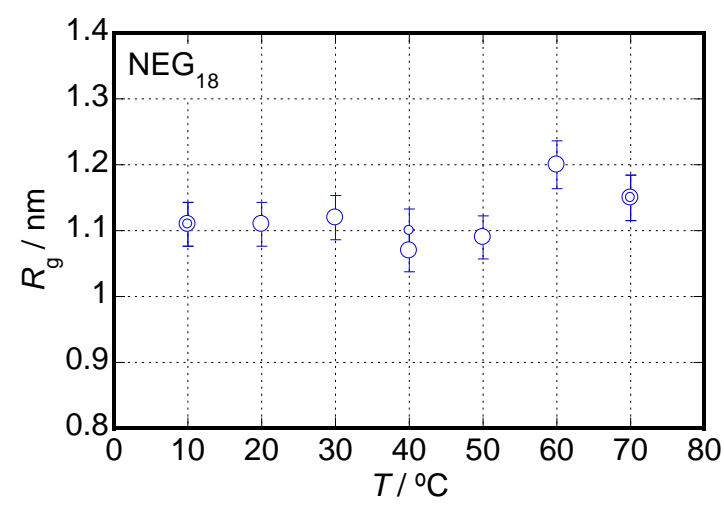

Figure 5 


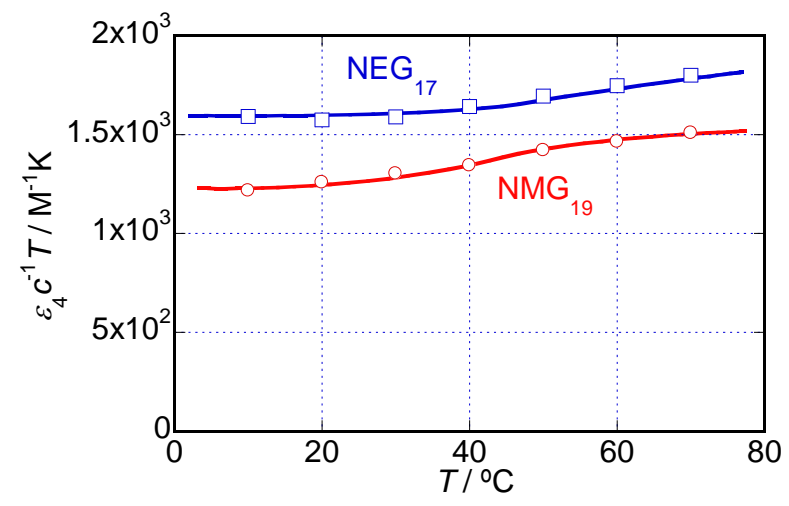

Figure 6
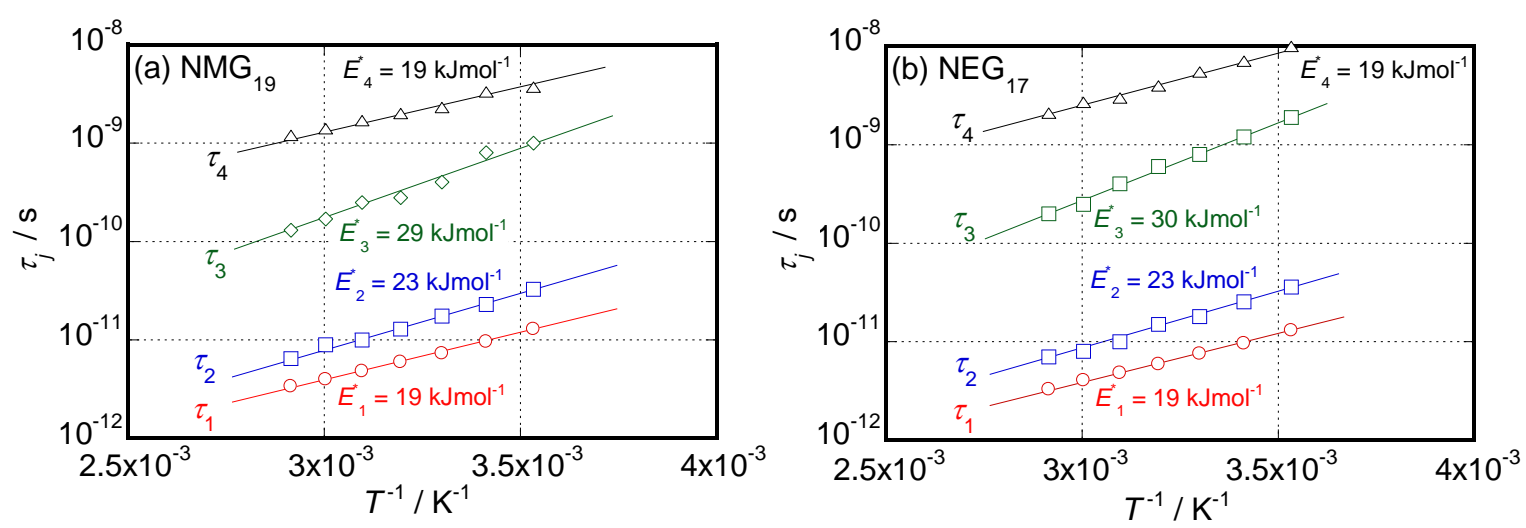

Figure 7 


\section{Pronounced Dielectric and Hydration Behaviors of}

\section{Monopolar Poly( $N$-alkylglycine)s in Aqueous Solution}

Kengo Arai ${ }^{1}$, Naoya Sagawa ${ }^{2}$, Toshiyuki Shikata ${ }^{* 1,2}$, Garrett L. Sternhagen ${ }^{3}$, Xin Li $^{3}{ }^{3}$ Li Guo, ${ }^{4}$ Changwoo Do ${ }^{5}$ and Donghui Zhang ${ }^{3}$

${ }^{1}$ Division of Natural Resources and Eco-materials, Graduate School of Agriculture, Tokyo University of Agriculture and Technology, 3-5-8 Saiwai-cho, Fuchu, Tokyo 183-8509, Japan

${ }^{2}$ Department of Symbiotic Science of Environment and Natural Resources, The United Graduate School of Agriculture, Tokyo University of Agriculture and Technology, 3-5-8 Saiwai-cho, Fuchu, Tokyo 183-8509, Japan

${ }^{3}$ Department of chemistry, Louisiana State University, Baton Rouge, Louisiana 70873, USA

${ }^{4}$ The Molecular Foundry, Lawrence Berkeley National laboratory, Berkeley, California 94720, USA

${ }^{5}$ Biology and Soft Matter Division, Neutron Sciences Directorate, Oak Ridge National Laboratory, Oak Ridge, TN 37831, USA

Monopolar Polypeptides: $\mathrm{NMG}_{19}$ and $\mathrm{NEG}_{17}$

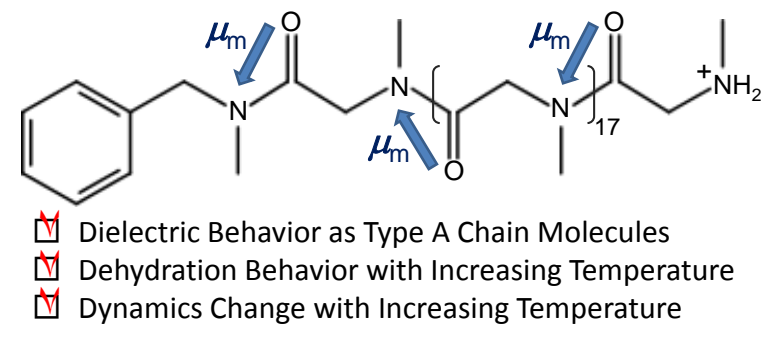

